\title{
COHERENT STATES IN FINITE QUANTUM MECHANICS ${ }^{+}$
}

\author{
G.G. Athanasiu*) and E.G. Floratos**) \\ Physics Dept. Univ. of Crete \\ and FO.R.T.H. \\ Heraklion-Crete \\ Greece

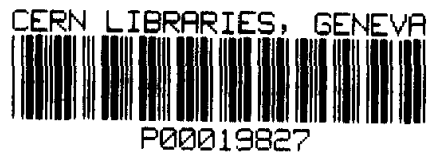

\begin{abstract}
The "harmonic oscillator", introduced by Itzykson and Balian, is considered, on finite phase-space lattices with the structure of Galois fields GF[p2]. We study this system at the classical and quantum mechanical level. Explicit construction of the quantum eigenstates provides us with a detailed understanding of the spectrum of scales relevant to this model. We construct also the coherent states appropriate to the finite Heisenberg-Weyl and Affine groups. Possible applications are discussed.
\end{abstract}

+) Work partially supported by the EEC grants, SCI-430C, CHRX-CT92-0063

*) eml : ATHANASI @ IESL.FORTH.GR

**) eml : FLORATOS @ IESL.FORTH.GR 


\section{Introduction}

Possible relevance of number theory in todays Physical theories, has been recently discussed in various contexts, ranging from discrete dynamical chaotic systems to field and string theories of fundamental particle interactions [1-5].

Also, introduction of quantum mechanical ideas, such as coherent states and representations of symmetries in the area of discrete algorithms for signal and image processing have recently attracted interest in the Physics Community [6]. So it seems to be an interesting problem the understanding of Quantum Mechanics and Quantum Field Theory on lattices over finite Galois fields [7].

Quantum Mechanics on a finite lattice, (FQM), was introduced by $\mathrm{H}$. Weyl, [8] and later on it was discussed in detail by J. Schwinger [9].Applications of (FQM) to the physics of the Quantum Hall (QH) effect started in the work of J. Zak and continued recently by J. Bellissard and collaborators [10], where the connection of QH effect with non-commutative geometry was emphasized [11].

In this paper we examine $\mathrm{FQM}$ of one degree of freedom over the simplest Galois field GF [p], [7]. We consider in detail, the classical and quantum "harmonic oscillator" introduced by R. Balian and C. Itzykson (B-I) [12]. In chapter 1 we discuss aspects of Classical Mechanics of discrete maps on the Galois field GF $\left[\mathrm{p}^{2}\right]$ regarded as a finite and discrete phase space, GF[p] x GF[p]. In chapters 2 and 3 we describe the explicit construction of the eigenstates and eigenvalues of the B-I oscillator. In chapter 4 we present the coherent states of the finite Heisenberg-Weyl group (Gabor-states) and in chapter 5 we introduce the coherent states of the extended finite Affine group $\mathrm{SL}(2, \mathrm{p}) / \mathrm{O}_{\mathrm{p}}(2)$ and discuss their properties. We conclude in chapter 6 with the discussion of open problems and possible applications. Finally in the Appendix, we collect for completeness, some elementary facts needed from number theory and the representation theory of the finite group SL $(2, \mathrm{p})[13,14]$. 
transformations (1.2-1.3) forms a finite group over GF [p], the special linear (or symplectic) group SL $(2, p)$ [12-14]. One choice for the generators of this group is :

$$
\mathrm{g}_{1}=\left(\begin{array}{ll}
1 & 1 \\
0 & 1
\end{array}\right), \quad \mathrm{g}_{2}=\left(\begin{array}{cc}
\mathrm{g} & 0 \\
0 & \mathrm{~g}^{-1}
\end{array}\right), \quad \mathrm{g}_{3}=\left(\begin{array}{cc}
0 & -1 \\
1 & 0
\end{array}\right)
$$

where $g$, is a primitive element of the multiplicative group $\mathrm{GF}^{*}[\mathrm{p}]=\mathrm{GF}[\mathrm{p}]-\{0\}$, [7]. There is in the place of $g_{3}$, another choice, which is a primitive or generating element, $\mathrm{R}_{0}$, for the abelian subgroup $\mathrm{O}_{\mathrm{p}}(2)$, of SL $(2, p)$ of matrices commuting with $g_{3}$ [12].

$$
\begin{aligned}
\mathrm{O}_{\mathrm{p}}^{(2)} & \equiv\left\{\left(\begin{array}{cc}
\mathrm{a} & \mathrm{b} \\
-\mathrm{b} & \mathrm{a}
\end{array}\right) \mid \mathrm{a}^{2}+\mathrm{b}^{2}=1 \bmod \mathrm{p}\right\} \\
& =\left\{\mathrm{R}_{0}, \mathrm{R}_{0}^{2}, \ldots, \mathrm{R}_{0}^{4 \mathrm{k}}\right\}
\end{aligned}
$$

Every primitive element $R_{0}$, has period $4 k$, for $p=4 k \pm 1$, the order of $O_{p}(2)$. For some well known facts about Galois fields relevant to our discussion see the appendix. The way to determine either of the primitive elements $g$ of $G F^{*}[p]$ or $R_{0}$ of $O_{p}(2)$ is by random search. For all prime numbers $p \leq 5.000$ the smallest primitive elements $g$ are tabulated by Abramowitz and Stegun, [17], while all the primitive elements of all primes $\leq 5.000$ are tabulated in ref. [18]. In ref. [19] a search was made for the generator $R_{0}$, of the "rotation" matrices mod $p$, for primes of the form $p=4 k-1$ in the range $p \leq 20.000$. In Table I we give $g$ and $R_{0}$ for $G F[p], p=4 k-1 \leq 2003$. For primes $p$ of the form $p=4 k+1$ it is possible to determine $R_{0}$ once a primitive element $g$ of $G F \cdot\{4 k+1]$ is known [see the appendix].

Coming back to the interpretation of the classical motion of the mappings $g_{1}, g_{2}, g_{3}$, or $\mathrm{R}_{0}(1.5,1.6)$ the first represents free motion, the second hyperbolic and the third, $\mathrm{R}_{0}$, "Oscillatory" motion with periods $\mathrm{p}, \mathrm{p}-1$ and $4 \mathrm{k}$ where $\mathrm{p}=4 \mathrm{k} \pm 1,[4,12]$. The periods of elements in SL $(2, p)$ must divide the order of the group, which is $p\left(p^{2}-1\right)$, so they must divide $\mathrm{p}$ or $\mathrm{p}-1$ or $\mathrm{p}+1[4]$. 


\section{Ch.2. The quantum B-I oscillator}

In a famous paper $\mathrm{A}$. Weil discovered the so called metaplectic representation of the symplectic group [20]. The basic idea, as far physics is concerned, is that since the representations of Heisenberg-Weyl, (HW), group are unique for a fixed value of the Planck constant $h$, if we transform the phase-space (position, momentum) parameters of the $\mathrm{H}$-W group by a symplectic transformation, we get another representation of this group for the same value of $h$ and so this must be an equivalent representation to the original one. The similarity unitary matrix can then define an irrep of the symplectic group.

For the finite and discrete phase-space $\mathrm{T}_{\mathrm{p}}^{2}$ this representation has been discussed in ref. $[12,15]$.

We start by constructing first the representations of the discrete and finite Heisenberg-Weyl group [8-10,12]. The configuration space of $T_{p}$ is the discrete circle, $\mathrm{GF}[\mathrm{p}]=\{0,1, \ldots, \mathrm{p}-1\}$, and so a basis for the position eigenfunctions is :

$$
1 \mathrm{q}>_{\mathrm{k}}=\delta_{\mathrm{kq}} \quad, \mathrm{q}=1,2, \ldots, \mathrm{p}
$$

The translation operator $\mathrm{P}$, which shifts by one position the states $(2.1)$ is :

$$
P=\left(\begin{array}{cccc}
0 & 0 & \ldots & 1 \\
1 & 0 & \ldots & 0 \\
0 & 1 & \ldots & 0 \\
\ldots & \ldots & 1 & 0
\end{array}\right)
$$

and its finite Fourier transform

$$
\mathrm{FP} \mathrm{F}^{-1}=\mathrm{Q} \text {, }
$$

where 


$$
J_{r, s} J_{r^{\prime}, s^{\prime}}=\omega^{\frac{r^{\prime} s-r s^{\prime}}{2}} J_{r+r^{\prime}, s+s^{\prime}}
$$

The metaplectic representation of $\operatorname{SL}(2, p)$ is defined through matrices $U(A)$ :

$$
\begin{gathered}
U^{-1}(A) J_{r, s} U(A)=J_{r^{\prime}, s^{\prime}} \\
\left(r^{\prime} s^{\prime}\right)=(r, s) A, \quad \forall A \in \operatorname{SL}(2, p)
\end{gathered}
$$

In [12] R. Balian and C. Itzykson presented an explicit form of the metaplectic representation $(2.9),[4,13,14]$ :

$$
U(A)=\frac{\sigma(1) \sigma(\delta)}{p} \sum_{r, s=0}^{\mathrm{p}-1} \omega^{\left[b r^{2}+(d-a) r s-c s^{2}\right] / 2 \delta} J_{r, s}
$$

where $A=\left(\begin{array}{ll}a & b \\ c & d\end{array}\right), \delta=2-a-d$ and

$$
\sigma(a)=\frac{1}{\sqrt{p}} \sum_{r=0}^{\mathrm{p}-1} \omega^{a r^{2}}=(a l p)\left\{\begin{array}{l}
1 \\
i
\end{array}\right\} \begin{aligned}
& \mathrm{p}=4 \mathrm{k}+1 \\
& \mathrm{p}=4 \mathrm{k}-1
\end{aligned}
$$

is the famous Gauss sum [7,21] while (alp) is \pm 1 , depending on whether a is, (or is not), the square of an integer mod $p$ (Jacobi symbol [7]).

The representation (2.10) is valid for $\delta \neq 0$ while when $\delta=0$ there are the following 

The characters of the $U_{L, R}$ representations are given in detail in [12]. The complete set of representations for SL $\left(2, G F\left[p^{n}\right]\right)$ is described in [13], [14]. It is possible to find explicitly the matrix elements of $U(A)$, for every $A \in S L(2, p)$, using the fact that :

$$
\left(J_{r, s}\right)_{k, 1}=\delta_{k-r, 1} \omega^{\frac{k+1-2}{2}} \cdot S
$$

and making repeated use of the Gauss sum (2.11):

$$
U_{k, l}(A)=\frac{1}{\sqrt{p}}(-2 c l p)\left\{\begin{array}{c}
1 \\
-i
\end{array}\right\} \cdot \omega^{-\frac{1}{2 c}}\left[a(k-1)^{2}+\alpha(l-1)^{2}-2(k-1)(1-1)\right]
$$

where the bracket symbol distinguished between $p=4 k+1,\{1\}$ and $p=4 k-1,\{-i\}$, cases.

Our interest is in studying the eigenvalues and eigenvectors of the generator $U\left(R_{0}\right)$, where $R_{0}$ is the generator of Op (2). For $p=4 k+1$ the unitary matrix which represents the scaling map, see (1.9):

$$
U_{k l}\left[\begin{array}{cc}
g & 0 \\
0 & g^{-1}
\end{array}\right]=\sigma(1) \sigma(\delta) \delta_{(k-1), g(l-1)}
$$

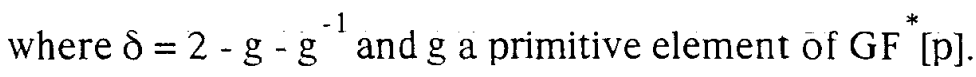

For $p=4 k-1$ the generic form of $G=U\left(R_{0}\right)$ is :

$$
G_{k, l}=\frac{1}{\sqrt{p}} i\left(2 b_{0} p\right) \cdot \omega^{\frac{1}{2 b_{0}}\left[a_{0}(k-1)^{2}+a_{0}(l-1)^{2}-2(k-1)(l-1)\right]}, R_{0}=\left(\begin{array}{cc}
a_{0} & b_{0} \\
-b_{0} & a_{0}
\end{array}\right)
$$




\section{Ch.1. Galois Phase Spaces}

Consider a particle moving on a discrete circle with finite number $\mathrm{N}$ of equidistand points. The classical phase space of this particle is a toroidal two dimensional finite lattice NxN, which in appropriate units can be labelled by the pairs of integers, [15],

$$
\mathrm{T}_{\mathrm{N}}^{2}=\{(\mathrm{m}, \mathrm{n}) \mid \mathrm{m}, \mathrm{n}=0,1,2, \ldots, \mathrm{N}-1\}
$$

Classical linear canonical transformations of this phase-space lattice, are $2 \times 2$ integer matrices with matrix multiplication mod $\mathrm{N}$. These matrices represent one-time step evolution maps, of Hamiltonians quadratic in phase-space variables $[4,5]$.

$$
\left(\begin{array}{c}
m_{t+1} \\
n_{t+1}
\end{array}\right)=\left(\begin{array}{ll}
a & b \\
c & d
\end{array}\right)\left(\begin{array}{c}
m_{t} \\
n_{t}
\end{array}\right) \quad t=0,1,2, \ldots
$$

where $m_{t}, n_{t}$ are the position and momentum of the particle at time step $t$ and $a, b, c, d$ are integers mod $\mathrm{N}$. To get Hamiltonian evolution we must preserve phase-space area $\bmod N$ :

$$
\operatorname{det}\left(\begin{array}{ll}
a & b \\
c & d
\end{array}\right)=1 \bmod N
$$

The restriction we have from doing all the arithmetic operations between integers mod $\mathrm{N}$ in a unique way, implies that $\mathrm{N}$ must be a prime number. For example the inverse of an integer mod $\mathrm{N}$ is unique if and only if $\mathrm{N}$ is a prime number [7]. Thus, the set of all integers

$$
\mathrm{GF}[\mathrm{p}]=\{0,1,2, \ldots, \mathrm{p}-1\}
$$

is closed under the four arithmetic operations. This is the simplest of the finite Galois fields GF $\left[\mathrm{p}^{\mathrm{n}}\right]$.

When the number of lattice points is a prime number, the set of all linear canonical 


\section{Ch.3. Eigenstates and eigenvalues of the B-I Quantum oscillator}

In [12] a method of construction was given, for the eigenstates of the Quantum B-I oscillator, through projector operators. Below we present a compact method, to construct the diagonalizing matrix of the Quantum B-I oscillator matrix G (ch.2). The period of matrix $G$ is $4 k$ for $p=4 k \pm 1$. Since $G$ is unitary the eigenvalues are:

$$
\lambda_{r}=e^{\frac{2 \pi i}{4 k} r}, \quad-m \leq r \leq m \quad m=\left\{\begin{array}{ll}
2 k-1, & p=4 k-1 \\
2 k, & p=4 k+1
\end{array}\right\}
$$

The ground state of the B-I oscillator corresponds to the eigenvalue $\mathrm{r}=0$ (no-nodes).

We can construct the unitary matrix of the eigenvectors of $G$ as the interwinner of $G$ and $\mathrm{D}_{\mathrm{G}}$ :

$$
\mathrm{V}=\frac{1}{4 k} \sum_{m=0}^{4 k-1} G^{m} D_{G}^{-m}
$$

Indeed we easily check

$$
\mathrm{G} \cdot \mathrm{V}=\mathrm{V} \cdot \mathrm{D}_{\mathrm{G}}
$$

that is, the column $r$ of the matrix $V$ is the orthonormalized eigenvector of $G,\left|\Psi_{r-2 k}\right\rangle$, corresponding to the eigenvalue $\lambda_{r-2 k}$.

$$
\left\langle\mathrm{q} \Psi_{\mathrm{r}-2 \mathrm{k}}\right\rangle=\frac{1}{4 \mathrm{k}} \sum_{\mathrm{m}=0}^{4 \mathrm{k}-1} \lambda_{\mathrm{r}-2 \mathrm{k}}^{-\mathrm{m}}(\mathrm{G})_{\mathrm{qr}}
$$

To construct the matrix power $G^{n}=U\left(R_{0}^{n}\right)$, we have to find the $2 \times 2$ matrix $R_{0}^{m}$, mod $p$ 
In this work we mainly study the Quantum Mechanics of $\mathrm{R}_{0}$, the "harmonic oscillator" of Balian and Itzykson (B-I) [12]. According to the results in the appendix, in the case $\mathrm{p}=4 \mathrm{k}+1, \mathrm{R}_{0}$ is essentially the scaling generator $\mathrm{g}_{2},(1.5)$, while for $\mathrm{p}=4 \mathrm{k}-1$ one has "true" rotations with "complex" eigenvalues $a_{0} \pm i b_{0}$. These belong to the quadratic extension of GF [p], [16],

$$
G F\left[p^{2}\right]=\left\{a+i b \mid a, b \in G F[p], i^{2}+1=0\right\} .
$$

on which $\mathrm{O}_{\mathrm{p}}(2)$ acts by multiplication

$$
\left(\begin{array}{ll}
a & b \\
-b & a
\end{array}\right)\left(\begin{array}{l}
x \\
y
\end{array}\right) \rightarrow(a+i b)(x+i y)
$$

The generator $\mathrm{R}_{0}$ of $\mathrm{O}_{\mathrm{p}}(2)$,can be considered as the unit time step map in the phasespace $T_{p}$, (1.1) corresponding to a harmonic oscillator in an external magnetic field [4], which from now on we shall call the B-I oscillator. 
$\mathrm{GF}^{*}[4 \mathrm{k}+1]$. Thus,

$$
R_{0}=\left(\begin{array}{ll}
\frac{g+g^{-1}}{2} & \frac{g^{-1}-g}{2 t} \\
\frac{g-g^{-1}}{2 t} & \frac{g+g^{-1}}{2}
\end{array}\right)
$$

and

$$
G=U\left(R_{0}\right)=U(T) U\left(\begin{array}{cc}
g & 0 \\
0 & g^{-1}
\end{array}\right) \quad U^{-1}(T)
$$

If the diagonalizing matrix of $U_{g}=U\left(\begin{array}{cc}g & 0 \\ 0 & g^{-1}\end{array}\right)$ is denoted by $V_{g}$ and its diagonal form $D_{g}$ :

$$
\mathrm{U}_{\mathrm{g}} \mathrm{V}_{\mathrm{g}}=\mathrm{V}_{\mathrm{g}} \mathrm{D}_{\mathrm{g}}
$$

then from (3.11) we get

$$
\mathrm{G} U(\mathrm{~T}) \cdot \mathrm{V}_{\mathrm{g}}=\mathrm{U}(\mathrm{T}) \mathrm{V}_{\mathrm{g}} \cdot \mathrm{D}_{\mathrm{g}}
$$

or $U(T) V_{g}$ is the unitary matrix of eigenvectors of $G$ and $D_{g}$ is at the same time, the diagonal form of $G$. The scaling matrix $U_{g}$ has matrix elements (rel. (2.20)) : 


$$
F_{k l}=\frac{1}{\sqrt{p}} \omega^{(k-1)(l-1)}, \quad k, l=1, \ldots, p
$$

is the exponential position operator

$$
Q=\left(\begin{array}{cccc}
1 & & & \\
& \omega & & \\
& & \cdots & \\
& & & \omega^{\mathrm{p}-1}
\end{array}\right), \omega=\mathrm{e}^{2 \pi \mathrm{i} / \mathrm{p}}
$$

The canonical Heisenberg commutation relations, in terms of $Q$ and $P$, become,

$$
\mathrm{QP}=\omega \mathrm{PQ}
$$

The general element of the $\mathrm{H}-\mathrm{W}$ group $[10,12,15]$ is given by :

$$
\mathrm{g}_{\mathrm{t}, \mathrm{r}, \mathrm{s}}=\omega^{\mathrm{t}} \mathrm{J}_{\mathrm{r}, \mathrm{s}} \equiv \omega^{\mathrm{t}+\frac{\mathrm{rs}}{2}} \mathrm{P}^{\mathrm{r}} \mathrm{Q}^{\mathrm{s}} \quad \mathrm{t}, \mathrm{r}, \mathrm{s}=0,1, \ldots, \mathrm{p}-1
$$

The $\mathrm{H}-\mathrm{W}$ group elements $\mathrm{J}_{\mathrm{r}, \mathrm{s}}$ realize a projective representation of the translation group in the phase-space $T_{p}^{2}[10]$, 
It is easy to check the action of the "circulant" scaling matrix $U_{g}(3.14)$, on the extended characters $\Pi_{j}, j=1, \ldots, p$ :

$$
U_{g} \Pi_{j}=-e^{-\frac{2 \pi j}{p-l} j} \Pi_{j}
$$

So the eigenvalue $(-1)$ appears twice $j=p-1, j=p=0$, while the ground state $j=\frac{p-1}{2}$ is the quadratic character

$$
10>=\prod_{\frac{p-1}{2}(a)}=\left(\begin{array}{c}
0 \\
\text { (alp })
\end{array}\right) \quad a=1, \ldots, p-1
$$

where (alp) is the Jacobi symbol [7].

The matrix $\mathrm{C}_{\mathrm{g}}$ is the eigenvector matrix $\mathrm{V}_{\mathrm{g}}$ of $\mathrm{U}_{\mathrm{g}}$ we were looking for. From rel. (3.13) we check that the matrix

$$
\mathrm{V}_{\mathrm{G}}=\mathrm{U}(\mathrm{T}) \cdot \mathrm{C}_{\mathrm{g}}
$$

is the eigenvector matrix of the B-I oscillator $G$ for $p=4 k+1$. The eigenvalue matrix $D_{g}$ is at the same time the eigenvalue matrix of $\mathrm{G}(3.2)$, but with some different order for the eigenvalues.

Since G commutes with F, the finite Fourier transform (FFT) (2.4), the eigenvectors of $\mathrm{G}$ are eigenvectors of $\mathrm{F}$ with eigenvalues (2.22) :

$$
f_{j}=i^{n}(-1)^{k} \cdot \lambda_{j}^{k}, \quad j=1,2, \ldots, p
$$

$\mathrm{n}=0,1$ for $\mathrm{p}=4 \mathrm{k} \pm 1$

These eigenvectors split the degeneracies of the FFT eigenvalues [12, 21]. 
particular cases [12] :

$$
\begin{gathered}
\delta=0, \quad b \neq 0 \quad U(A)=\frac{\sigma(-2 b)}{\sqrt{p}} \sum_{r=0}^{p-1} \omega^{r^{2} / 2 b} J_{\frac{r(a-1)}{b}, r} \\
\delta=b=0, c \neq 0 \quad U(A)=\frac{\sigma(-2 c)}{\sqrt{p}} \sum_{r=0}^{p-1} \omega^{-r^{2} / 2 c} P^{r} \cdot \\
U(I)=I_{p x p}
\end{gathered}
$$

The representation of $\left(\begin{array}{cc}0 & -1 \\ 1 & 0\end{array}\right)$ has as the finite Fourier transform (FFT):

$$
U\left(\begin{array}{cc}
0 & -1 \\
1 & 0
\end{array}\right)=(-1)^{k+1} i^{n} F
$$

where $\mathrm{n}=0,1$ according to if, $\mathrm{p}=4 \mathrm{k}+1$ or $\mathrm{p}=4 \mathrm{k}-1$.

The matrix $U(A)$ commutes with the square of FFT which is the parity operator,

$$
S=F^{2}, \quad S l q>=1-q>, \quad q=1,2, \ldots, p
$$

So we have the left-mover and the right-mover representations of the H-W group, [12].

$$
\mathrm{U}_{\mathrm{L}}=\mathrm{U}(\mathrm{I}+\mathrm{S}) / 2, \quad \mathrm{U}_{\mathrm{R}}=\mathrm{U}(\mathrm{I}-\mathrm{S}) / 2
$$


$\frac{1}{\mathrm{~N}} \underset{\overrightarrow{\mathrm{m}}}{ } \mid \overrightarrow{\mathrm{m}}><\overrightarrow{\mathrm{m} \mid}=\mathrm{I}$

because the left hand side (lhs), commutes with all the $\mathrm{J}_{\vec{n}}$ 's and the irreducibility of the $\mathrm{H}-\mathrm{W}$ group representation implies that the $\mathrm{l}$ s is a scalar number, times the identity matrix. We normalize the sum by

$$
\left.\mathrm{N}=\sum_{\overrightarrow{\mathrm{m}}}|\overrightarrow{\mathrm{n}}| \overrightarrow{\mathrm{m}}\right\rangle\left.\right|^{2}
$$

The coherent states, eventhough normalized, are not orthogonal :

$$
\left.\langle\vec{n} \mid \vec{m}\rangle=\omega^{\frac{\vec{m} \times \vec{n}}{2}}<0\left|J_{m-n}\right| 0\right\rangle
$$

where $\vec{m} \times \vec{n}=m_{1} n_{2}-m_{2} n_{1}$.

The overlap constants $\langle\vec{n} \mid \vec{m}\rangle$ are constant on the $R_{0}$ orbits :

$$
\langle\vec{n} \mid \vec{m}\rangle=\left\langle\vec{n} R_{0}^{-1} \mid \vec{m} R_{0}^{-1}\right\rangle
$$

Finally the matrices $\overrightarrow{\mathrm{m}}$ on the coherent states realize a projective representation [23] of the $T_{2}$ translation group of $G F\left[p^{2}\right]$.

$$
J \vec{m} \mid \vec{n}>=\omega^{\frac{\vec{n} \times \vec{m}}{2}} \vec{n}+\vec{m}>
$$

The $\mathrm{p}^{2}$ coherent states are linearly dependent and the system is overcomplete. 
$-10-$

Notice also the relations [12] :

$$
F=i^{n}(-G)^{k}, \quad S=(-1)^{n} G^{2 k}
$$

$\mathrm{n}=0,1$ if $\mathrm{p}=4 \mathrm{k} \pm 1$ respectively. 


$$
\overrightarrow{\mathrm{m}}>=\mathrm{J}_{\mathrm{m}} \mathrm{U}(\mathrm{T}) \Pi_{\frac{\mathrm{p}-1}{2}}
$$

or using the definition of the metaplectic representation :

$$
\overrightarrow{\mathrm{m}}>=\mathrm{U}(\mathrm{T}) \underset{\mathrm{m} \cdot \mathrm{T}}{\overrightarrow{\mathrm{m}}} \Pi_{\frac{\mathrm{p}-1}{2}}
$$

where $\vec{m}=\left(m_{1}, m_{2}\right)$,

$$
\overrightarrow{\mathrm{m}} \cdot \mathrm{T}=\frac{1}{1+\mathrm{t}}\left(\mathrm{m}_{1}-\mathrm{tm}_{2}, \mathrm{~m}_{1}+\mathrm{tm} 2\right) \equiv\left(\mathrm{m}_{1}^{\mathrm{T}}, \mathrm{m}_{2}^{\mathrm{T}}\right)
$$

The position representation of $\overrightarrow{\mathrm{m}}>$ can be expressed in terms of hybrid Gauss sums with Jacobi symbols as coefficients. To get an easier representation we pass over the modified coherent states

$$
\overrightarrow{\mathrm{Im}}>_{\mathrm{T}}=\mathrm{J}_{\mathrm{m}} \cdot \mathrm{T} T \frac{\mathrm{p}-\mathrm{l}}{2}
$$

In this basis where $\mathrm{G}$ is represented by a circulant matrix, rel. (3.14), we obtain :

$$
\langle\mathrm{r} \mid \overrightarrow{\mathrm{m}}\rangle_{\mathrm{T}}=\frac{1}{\sqrt{\mathrm{p}-1}}\left(\mathrm{r}-\mathrm{m}_{1}^{\mathrm{T}}-1 \mid \mathrm{p}\right) \omega^{\frac{2(\mathrm{r}-1)-\mathrm{m}_{1}^{\mathrm{T}}}{2}} \cdot \mathrm{m}_{2}^{\mathrm{T}}
$$

and $\mathrm{m}_{1}^{\mathrm{T}}, \mathrm{m}_{2}^{\mathrm{T}}$ are given in rel. (4.15). The overlap constants $\langle\overrightarrow{\mathrm{n}} \mid \overrightarrow{\mathrm{m}}\rangle$ are the same :

$$
\left\langle\langle\vec{n} \mid \vec{m}\rangle_{T}=\langle\vec{n} \mid \vec{m}\rangle .\right.
$$




$$
\begin{aligned}
& R_{0}^{n}=\left(\begin{array}{cc}
a_{0} & b_{0} \\
-b_{0} & a_{0}
\end{array}\right)^{n} \equiv\left(\begin{array}{cc}
a_{n-1} & b_{n-1} \\
-b_{n-1} & a_{n-1}
\end{array}\right), \quad n=, 1, \ldots, 4 k-1 \\
& R_{0}^{0}=\left(\begin{array}{cc}
a_{-1} & b_{-1} \\
-b_{-1} & a_{-1}
\end{array}\right)=\left(\begin{array}{ll}
1 & 0 \\
0 & 1
\end{array}\right)
\end{aligned}
$$

and then to use rel. (2.19). We find that the eigenvectors (3.5) are $\left(\mathrm{a}_{-1}=\mathrm{b}_{-1}=0\right)$;

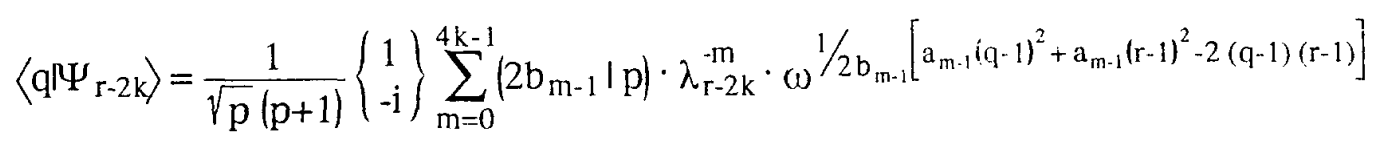

These sums are the so called Kloosterman generalized sums in number theory and oniy very specific cases can be expressed in closed form [16].

In the case $\mathrm{p}=4 \mathrm{k}+1$ it is possible to find explicit closed expressions for the eigenstates of the B-I oscillator in a particular basis. As we discuss in the appendix, in this case, the $O_{p}(2)$ generator $R_{0}$ is similar to the scaling map, $\left(t^{2}=-1\right.$ modp) :

$$
\left(\begin{array}{cc}
a & b \\
-b & a
\end{array}\right)=T\left(\begin{array}{cc}
a-t b & 0 \\
0 & a+t b
\end{array}\right) T^{-1}, a^{2}+b^{2}=1 \operatorname{modp}
$$

where the matrix

$$
\mathrm{T}=\frac{1}{\sqrt{2 \mathrm{t}}}\left(\begin{array}{cc}
1 & 1 \\
-\mathrm{t} & \mathrm{t}
\end{array}\right)
$$

belongs to $\operatorname{SL}(2, \mathrm{p})$ since $2 t=(1+t)^{2}$ and det $T=1$.

To find $R_{0}$ we choose $a_{0}-t b_{0}=g$ and so $a_{0}+t b_{0}=g-1$ where $g$ a primitive element of 
$(\mathrm{A} \mathrm{B})_{\mathrm{m}, \overrightarrow{\mathrm{n}}}=\frac{1}{\mathrm{~N}} \sum_{\overrightarrow{\mathrm{k}}} \mathrm{A}_{\mathrm{m}, \overrightarrow{\mathrm{k}}} \mathrm{B}_{\overrightarrow{\mathrm{k}}, \overrightarrow{\mathrm{n}}}$

and

$$
\begin{gathered}
\langle\overrightarrow{\mathrm{m}}|A| \psi\rangle=\frac{1}{N} \sum_{\vec{n}} A_{m, \vec{n}}\langle\vec{n} \mid \psi\rangle \\
\cdots \\
\left(U^{-1}\left(R_{0}\right) A U\left(R_{0}\right)\right)_{\vec{m}, \vec{n}}=A_{\vec{m} R_{0} \vec{n} R_{0}}
\end{gathered}
$$

We close this section by noticing that, we can get a representation with classical behaviour, for the transformation properties of any matrix A, under any element of $\operatorname{SL}(2, p)$ (ch. 2), if we first expand $A$ on the basis $\mathrm{J}_{\mathrm{m}}$ :

$$
A=\frac{1}{p} \sum_{\vec{m}} A_{m} \vec{m} J_{m}
$$

where

$$
A_{\vec{m}}=\operatorname{Tr}\left(A_{J} \overrightarrow{-m}\right)
$$

Indeed from ch.2 we get

$$
U^{-1}(R) A U(R)=\frac{1}{p} \sum_{\vec{m}} A_{m} \vec{J} J_{\vec{m}} \cdot R=\frac{1}{p} \sum_{\vec{m}} A_{\vec{m}} \cdot R^{-1} J_{m} \vec{m}
$$

So the representaion of $\mathrm{A}$ by the matrix 


$$
\mathrm{U}_{\mathrm{kl}}\left[\begin{array}{cc}
\mathrm{g} & 0 \\
0 & \mathrm{~g}^{-1}
\end{array}\right]=-\delta_{\mathrm{k}-1, \mathrm{~g}(1-1)}
$$

We find explicit forms of the eigenvectors of $U_{\mathrm{g}}$ by using the multiplicative characters of $\mathrm{GF}^{*}[\mathrm{p}],[16]$.

Relatively to the primitive element $g$ of $\mathrm{GF}^{*}[\mathrm{p}]$, the multiplicative characters are defined as the $\mathrm{p}-1$ functions on $\mathrm{GF}^{*}[\mathrm{p}]$ :

$$
\Pi_{j}(\mathrm{~g})=\mathrm{e}^{\frac{2 \pi \mathrm{i}}{\mathrm{p}-1} \mathrm{jn}} / \sqrt{\mathrm{p}-1} \quad \begin{gathered}
\mathrm{j}=1,2, \ldots, \mathrm{p}-1 \\
\mathrm{n}=1,2, \ldots, \mathrm{p}-1
\end{gathered}
$$

and the p-th function is defined as :

$$
\Pi_{\alpha}\left(g^{n}\right)=0, \quad n=1,2, \ldots, p 1
$$

We extend these characters also on the zero-element of GF[p] :

$$
\Pi_{0}(p)=1, \Pi_{j}(0)=0 \quad j=1, \ldots, p-1
$$

Thus we construct the extended pxp, character matrix $\mathrm{C}_{\mathrm{g}}$

$$
\mathrm{C}_{\mathrm{g}}=\left(\begin{array}{ccccc}
0 & 0 & \ldots & 0 & 1 \\
\Pi_{1} & \Pi_{2} & & \Pi_{\mathrm{p}-1} & 0 \\
& & & \cdot \\
& & & & 0
\end{array}\right)
$$


In the case of the discrete field $F=G F[p]$ [29] we can use our elementary matrices $P$ (2.2), and the circulant matrix, $D=D_{g}^{-1}(2.20)$, to contruct the general element $U(\lambda, \alpha)$ $(5.4)$,

$$
\begin{array}{ll}
{[P f](k)=f(k-1)} & k=0,1, \ldots, p-1 \\
{[D f](k)=f(g k)} & k=0,1, \ldots, p-1
\end{array}
$$

We check that

$$
\mathrm{U}(\lambda, \alpha)=\mathrm{D}^{1 / \lambda} \cdot \mathrm{p}^{-\alpha}
$$

The representation defined above is reducible and there is an invariant subspace of dimension one (the constant function) [30].

Here we shall construct generalized wavelets (we call them p-wavelets), on the discrete field GF[p], as coherent states paramerized by the set $\operatorname{SL}(2, p) / \mathrm{O}_{\mathrm{p}}(2)$. Every element $g \in \operatorname{SL}(2, p)$ can be factorized as a product of an $O_{p}(2)$ element $r(g)$, times another unique element $h(g)$

$$
g=h(g) r(g)
$$

Indeed let $g=\left(\begin{array}{ll}a & b \\ c & d\end{array}\right) \in \operatorname{SL}(2, p)$. If the vector $(c, d)$ has norm which is a square modulo $p$, (We choose here $p=4 k-1$. If $p=4 k+1$ we have an analogous argument),

$$
c^{2}+d^{2}=1^{2} \bmod p, \quad l \in G F^{*}[p]
$$

then we can find a unique relation $r(g)$ such that

$$
g \cdot r^{-1}(g)=\left(\begin{array}{ll}
a^{\prime} & b^{\prime} \\
0 & l
\end{array}\right)
$$




\section{Ch.4. Heisenberg - Weyl coherent states}

Coherent states are usually defined as quantum mechanical states, which evolve in time following as closely as possible the motion of a classical system, that is with the smallest possible dispersion. There is an extensive literature for coherent states in physics, [22, 23], but also recent applications to signal and image analysis are developing fast $[6,24]$. Coherent states in discrete rational phase-space lattices, (or Von-Neumann lattices), have been discussed in papers by $\mathrm{J}$. Zak and collaborators, which deal with the problem of quantum Hall effect $[10,25]$.

In ch. 3 we have determined the eigenstates of the B-I oscillator so we can proceed to define the corresponding coherent states over the Galois Field GF [p] :

$$
\left|\overrightarrow{\mathrm{m}}>=\mathrm{J}_{\mathrm{m}}\right| 0>, \quad \overrightarrow{\mathrm{m}}=\left(\mathrm{m}_{1}, \mathrm{~m}_{2}\right) \in \mathrm{GF}[\mathrm{p}] \times \mathrm{GF}[\mathrm{p}] \approx \mathrm{GF}\left[\mathrm{p}^{2}\right]
$$

where the coset elements of the H-W group over its U(1) center are [rel. (2.7)] :

$$
J_{\mathrm{m}}=\omega^{\frac{\mathrm{m}_{1} \mathrm{~m}_{2}}{2}} P^{\mathrm{m}_{1}} \mathrm{Q}^{\mathrm{m}_{2}} \quad \mathrm{~m}_{1}, \mathrm{~m}_{2}=, 1, \ldots, \mathrm{p}
$$

and $10>$ is the normalized ground state of the B-I oscillator. From the definition (2.21), we see that :

$$
\mathrm{U}\left(\mathrm{R}_{0}\right)|\overrightarrow{\mathrm{m}}>=| \overrightarrow{\mathrm{m}} \cdot \mathrm{R}_{0}^{-1}>
$$

which implies that the state $\vec{m}>$ "moves" classically under the action of $U\left(R_{0}\right)$. The coherent states just defined resolve the identity operator : 
In ref. [19] we show that always exists primitive element of $G F\left[p^{2}\right], w=w_{1}+i w_{2}$ which has norm:

$$
w_{1}^{2}+w_{2}^{2}=g
$$

From $(5.17),(5.18)$ it follows that we can find a rotation $r(g)$ such that

$$
(c, d)=\left(l w_{1}, l w_{2}\right) r(g)
$$

The decomposition of $\mathrm{g}$ in this case becomes

$$
\left(\begin{array}{ll}
a & b \\
c & d
\end{array}\right)=h(g) \cdot r(g)
$$

where,

$$
\begin{gathered}
h(g)=\left(\begin{array}{cc}
(\lg )^{-1} & 0 \\
0 & 1 g
\end{array}\right)\left(\begin{array}{cc}
1 & (a c+b d) g \\
0 & 1
\end{array}\right)\left(\begin{array}{cc}
w_{2} & -w_{1} \\
\frac{w_{1}}{g} & \frac{w_{1}}{g}
\end{array}\right) \\
r(g)=(l g)^{-1}\left(\begin{array}{cc}
c w_{1}+d w_{2} & d w_{1}-c w_{2} \\
-d w_{1}+c w_{2} & c w_{1}+d w_{2}
\end{array}\right)
\end{gathered}
$$

In both cases the non-rotational part of an element $\mathrm{g}$, in SL $(2, \mathrm{p})$, or its coset (SL (2, p) $/ \mathrm{O}_{\mathrm{p}}$,) representative, is labeled by the elements of $G F\left[\mathrm{p}^{2}\right]$ :

$$
z \equiv g \cdot i=\frac{a i+b}{c i+d}
$$

The transformation of cosets under a group multiplication $\mathrm{g}^{\prime}$ is given as :

$$
z^{\prime}=g^{\prime} \cdot z=\left(g^{\prime} \cdot g\right) \cdot i
$$




$$
\overrightarrow{\mathrm{n}}>=\frac{1}{\mathrm{~N}} \sum_{\overrightarrow{\mathrm{m}}} \overrightarrow{\mathrm{m}}><\overrightarrow{\mathrm{m}} \mid \overrightarrow{\mathrm{n}}>
$$

for all states $\mid \vec{n}>$.

Although the standard quantum mechanical coherent states, [22], are easily visualized as frequency modulated gaussians, which have been shifted in position and momentum space, their discrete and finite analogs, (4.1), have non-gaussian patterns. Below we shall discuss the case of Galois fields GF[p], where $p=4 k+1$. In this case, we found in ch.3 that the B-I oscillator is similar to the scaling operator and its eigenstates are the multiplicative characters of $\mathrm{GF}[\mathrm{p}]$ in an appropriate basis. From (3.9-3.20) we see that the eigenstates of $B-I$ oscillator for $p=4 k+1$ are :

$$
\mid \mathrm{j}>=\mathrm{U}(\mathrm{T}) \prod_{\frac{\mathrm{p}-1}{2} \cdot \mathrm{j}}, \quad \mathrm{j}=0,1,2, \ldots, \mathrm{p}-1
$$

and the generator $\mathrm{G}$ is similar to the scaling operator :

$$
G=U(T) U\left(\begin{array}{cc}
g & 0 \\
0 & g^{-1}
\end{array}\right) U^{-1}(T)
$$

where $g$ is a primitive element of $G F[p]$ and $\Pi_{j}$ are the multiplicative characters (rel. 3.15-3.17). The matrix $T \in \operatorname{SL}(2, p)$, is given by rel. (3.9),

$$
T=\frac{1}{1+t}\left(\begin{array}{cc}
1 & 1 \\
-t & t
\end{array}\right), \quad t^{2}=-1 \operatorname{modp}
$$

The coherent states for $\mathrm{p}=4 \mathrm{k}+1$ are defined as : 


\section{Ch. 6 Conclusions}

Although in the literature there is a lot of discussion, about p-adic Quantum Mechanics (QM), we think that examining in detail QM, over Galois fields is a separate issue which is worth studying from the point of view of mathematical completeness and structure as well as from the physics point of view.

Classical linear dynamical systems on toroidal phase spaces, exhibit "chaotic" behaviour, which can be controlled and studied also at the Quantum Mechanical level with smaller computational effort than the non-linear ones [4a, 5, 31, 32]. We consider our work as one more step in the discretization and "decomposition" of the linear dynamical systems on toroidal phase spaces.

We hope, we have showed that, the classical and quantum mechanical behaviour of discrete linear systems over Galois phase-spaces, (especially of the BI oscillator), can be studied in great detail with the help of elementary Number theory.

Tractable problems in our approach are extensions to other Galois fields, $G F\left[p^{n}\right]$, increasing the number of degrees of freedom or to formulate field and string theories over Galois fields. More fundamental problem seems to be the approach to the continuum and its stability.

The relation of classical and quantum behaviour also is of great interest and should be studied along the lines of reference [5].

Finally but not lastly the coherent state analysis of signals and images over Galois fields is an intriguing and challenging problem due to the intrinsic randomization introduced by the algebraic operations of these number fields.

\section{Acknowledgments}

We thank C. Itzykson, A. Voros and G. Savvidy, for useful discussions. 
We discuss finally some general properties of the coherent states $\vec{m}\rangle$. It is easy to see that the overlapping constants $\langle\vec{m} \mid \vec{n}\rangle$ define a reproducing Kernel

$$
\begin{gathered}
K(\vec{m} \mid \vec{n})=\langle\vec{m} \mid \vec{n}\rangle, \\
\frac{1}{N} \sum_{\vec{m}} K(\vec{i} \mid \vec{m}) K(\vec{m} \mid \vec{n})=K(\vec{i} \mid \vec{n})
\end{gathered}
$$

which acts on functions like a $\delta$-function :

$$
\frac{1}{N} \sum_{\vec{n}} K(\vec{m} \mid \vec{n}) \psi(\vec{n})=\psi(\vec{m})
$$

where

$$
|\psi\rangle=\frac{1}{N} \sum_{\vec{n}} \overrightarrow{\ln }>\psi(\vec{n})
$$

Also an arbitrary pxp matrix A, can be represented through its symbol [22] :

$$
\langle\overrightarrow{\mathrm{m}}|\mathrm{A}| \overrightarrow{\mathrm{n}}\rangle=\mathrm{A}_{\mathrm{m} \cdot \overrightarrow{\mathrm{n}}}
$$

which has the convolution property, 


$$
c=\left(\begin{array}{ll}
1 & 1 \\
0 & 1
\end{array}\right), \quad \mathrm{d}=\left(\begin{array}{ll}
1 & \mathrm{~g} \\
0 & 1
\end{array}\right)
$$

and $b$ finally is the generator $R_{0}$, of the $O_{p}(2)$ subgroup of $\operatorname{SL}(2, p)$ (rel. 1.6) of order $4 \mathrm{k}=\mathrm{p} \pm 1$.

The representations of are divided in two classes $[13,14]$. Those of the first class are constucted on the space of homogeneous functions on $\mathrm{G} \equiv \mathrm{GF}[\mathrm{p}] \times \mathrm{GF}[\mathrm{p}]$

for some multiplicative character $\pi$ of GF[p] and and those of the second class on the space of factorizable function on $\left.G F\left[p^{2}\right]=\{x+\sqrt{ } g y) \mid x, y, \in G F[p]\right\}$,

$$
\left.\begin{array}{l}
f(t \cdot z)=\pi(t) f(z), \quad \forall z^{\prime} E G F\left[p^{2}\right] \\
t^{\prime} E\left\{x+\sqrt{g} y \mid x^{2}-g y^{2}=1\right.
\end{array}\right\}
$$

and $\pi$ a character of the $O_{p}(2)$. One finally has in total, eight types of representations and the non trivial ones have dimensions, $q, q+1,1 / 2(q+1), q-1,1 / 2(q-1)$. The metaplectic representation which we studied is the unique of dimension $q$.

The elements of $\mathrm{GF}^{*}[\mathrm{p}]$ are either quadratic residues (squares) modp or not (both (p1) $/ 2$ in number). The function which separates these two, is the Legendre symbol $(n \mid p)=1$, $-1,0$ for qres, nqres, and $n=0$, respectively.

The Legendre symbol satisfies the famous quadratic reciprocity law

$$
(p \mid q)(q \mid p)=(-1)^{(p-1)(q-1) / 4}
$$

For example the numbers $-1,2$ are characterized as follows :

$$
\begin{aligned}
& (-1 / \mathrm{p})=(-1)^{(\mathrm{p}-1) / 2} \\
& (2 \mathrm{p})=(-1)^{\left(\mathrm{p}^{2}-1\right) / 8}
\end{aligned}
$$




$$
\begin{gathered}
-22- \\
J(A)=\left\{A_{m}\right\}_{m_{l}, m_{2}=1, \ldots p}
\end{gathered}
$$

follows the classical motion of its indices.

\section{Ch.5 Coherent states for the affine group $\mathrm{SL}(2, \mathrm{p}) / \mathrm{O}_{\mathrm{p}}(2)$}

The affine group, Aff (F), over a number field F, (which is taken to be here GF[p]), is defined as the group generated by translations and scalings on $\mathrm{F}$ :

$$
\mathrm{g}(\lambda, \alpha) \mathrm{x}=\lambda \mathrm{x}+\alpha \quad \forall \mathrm{x} \in \mathrm{F}, \quad \alpha \in \mathrm{F}, \quad \lambda \in \mathrm{F}-\{0\}
$$

The composition law follows to be,

$$
\mathrm{g}(\lambda, \alpha) \mathrm{g}\left(\lambda^{\prime}, \alpha^{\prime}\right)=\mathrm{g}\left(\lambda \lambda^{\prime}, \lambda \alpha^{\prime}+\alpha\right)
$$

and the inverse is,

$$
g^{-1}(\lambda, \alpha)=g\left(\lambda^{-1},-\alpha \lambda^{-1}\right)
$$

The representation theory of this $\operatorname{Aff}(R)$ group over the field of feal number is known [26] and coherent states corresponding to this group have been studied in [27, 28] (see also [22]).

- In signal and image processing, a new basis of functions (called wavelets) have been constructed which probe the spectrum of scales of signals at every position and the method is called multiresolution analysis (MRA) [6, 29]. Quite recently the method has been applied also to discrete fields [30]. In principle one uses the following representaion of Aff[F] on the space of complex functions on $F, C(F)$,

$$
[\mathrm{U}(\lambda,-\alpha) \mathrm{f}](\mathrm{x})=\mathrm{f}\left(\mathrm{g}^{-1}(\lambda, \alpha) \mathrm{x}\right), \quad \forall \mathrm{x} \in \mathrm{F} .
$$

Choosing appropriate discrete set of values of $\lambda_{n}$, (usually $\lambda_{n}=\lambda_{0} \cdot 2^{n}$ ), $\alpha_{m}$ and an initial function $\mathrm{f}$, a set of functions $\mathrm{f}_{\mathrm{nm}}$ is contructed through (5.4), which is at least complete in $\mathrm{C}(\mathrm{F})$. The MRA method describe the conditions under which, we can analyze on the wavelets a signal and how we can recontruct it from its wavelet components. 
in the case $p=4 \mathrm{k}+1$.

In this case the diagonalizing matrix of any element in $\mathrm{O}_{\mathrm{p}}(2)$ is :

$$
\left(\begin{array}{cc}
a & b \\
-b & a
\end{array}\right)=T\left(\begin{array}{cc}
a-t b & 0 \\
0 & a+t b
\end{array}\right) T^{-1}
$$

where

$$
\mathrm{T}=\frac{1}{\sqrt{2 \mathrm{t}}}\left(\begin{array}{cc}
1 & 1 \\
\mathrm{t} & -\mathrm{t}
\end{array}\right)
$$

and $t^{2}=-1 \bmod p$. We notice that $t=\left(\frac{p-1}{2}\right) !$ is a solution in GF[p] and that $2 t=(1+t)^{2}$, so $\sqrt{2 t}=1+t \in G F[p]$.

We choose

$$
a-t b=g, a+t b=g^{-1}
$$

where $g$ is a primitive element of GF[p] then

$$
a_{0}=\frac{g+g^{-1}}{2}, \quad b_{0}=\frac{g^{-1}-g}{2 t}
$$

form the elements of $R_{0}$

$$
R_{0}=\left(\begin{array}{cc}
a_{0} & b_{0} \\
-b_{0} & a_{0}
\end{array}\right)
$$

with minimum period $\mathrm{p}-1=4 \mathrm{k}$. 
We check easily that this geometrical contruction gives $r(g)$ and $h(g)$ :

$$
\left(\begin{array}{ll}
a & b \\
c & d
\end{array}\right)=\left(\begin{array}{cc}
1^{-1} & (a c+b d) \cdot l^{-1} \\
0 & 1
\end{array}\right)\left(\begin{array}{cc}
d \cdot l^{-1} & c \cdot l^{-1} \\
-c \cdot l^{-1} & d \cdot l^{-1}
\end{array}\right)=h(g) \cdot r(g)
$$

On the other hand $\mathrm{h}(\mathrm{g})$, is a product of scaling and translation operations

$$
h(g)=\left(\begin{array}{cc}
1^{-1} & 0 \\
0 & 1
\end{array}\right)\left(\begin{array}{cc}
1 & a c+b d \\
0 & 1
\end{array}\right)
$$

Acting on GF[p] as, (see ref. [28] for the continuum case, $\mathrm{SL}(2 \mathrm{R}) / \mathrm{O}(2)$ ),

$$
\left(\begin{array}{ll}
a & b \\
c & d
\end{array}\right) \cdot x=\frac{a x+b}{c x+d}, \quad x \neq-d / c \quad x \in G F[p],
$$

We find

$$
h(g) \cdot x=l^{-2}(x+a c+b d)
$$

If we denote by $i$ the square root of $-1 \in G F[p]$ and we extend in $G F\left[p^{2}\right]$ the action (5.13),

$$
g \cdot z=\left(\begin{array}{ll}
a & b \\
c & d
\end{array}\right) \cdot z=\frac{a z+b}{c z+d}, \quad z=x+i y \in G F\left[p^{2}\right]
$$

we can check that the real and imaginary parts of

$$
g \cdot i=h(g) \cdot i
$$

contain the two parameters of $h(g), 1$ and actbd. If on the other hand $c^{2}+d^{2}$ is not the square of an integer mod $p$, then we can find a primitive element $g$ of GF $[p]$, such that

$$
c^{2}+d^{2}=1^{2} \cdot g, \quad l \in G F^{*}[p]
$$


[12] R. Balian and C. Itzykson, C.R. Acad. S. Paris, t.303 Série I, no 16 (1986) 773.

[13] M. Gel'fand, M.I. Graev and I.I. Piatetskii-Shapiro, "Representation theory and automorphic functions" Saunders-London (1966, 1990).

[14] S. Tanaka, Osaka J. Math $\underline{4}$ (1967) 65; J.E. Humphreys, Am. Math. Monthly $\underline{82}$ (1975) 21.

[15] For a simplified discussion in another context see: E.G. Floratos, Physics Letters B228 (1989) 335 .

[16] B. Lidl and H. Niederreiter, "Finite Fields", Vol. 20, Encyclopedia of Mathematics and its applications. Cambridge Un. Press 1984.

[17] M. Abramowitz and I. Stegun, "Handbook of Mathematical Functions" N.B.S., Washington 1964.

[18] H. Hanptman, E. Vegh and J. Fisher "Tables of All primitive roots for primes less than 5000", NRL Report 7070, 1970, Washington D.C. For further informatin see ref. [16].

[19] G. G. Athanasiu and E.G. Floratos, "Polar decomposition of primitive elements in GF $\left[p^{2}\right]$ ", Crete Univ. Preprint, CRETE.TH/9/93.

[20] A. Weil, "Sur certain groupes d'operateurs unitaires", Acta Math. 111 (1964) 143211.

[21] L. Auslander and P. Tolimieri, Bull. Amer. Math. Soc. 1, No6 91979) 847; M.L. Mehta "Matrix Theory" Les Edition de Physique, 1989.

[22] A. Perelomov "Generalized Coherent States and their applications", Springer Verlag 1986.

[23] J.R. Klauder and B-S Skagerstam, "Coherent States", World Scientific 1985.

[24] S.G. Mallat, IEEE Trans. Acous. Speech and Signal Proc. Vol. $\underline{37}$ (1989) 2091.

[25] P. Wiegmann, Seminar in ENS Summer school, Paris 1993. 


$$
-26-
$$

We define now the p-wavelets as coherent states based on the $\mathrm{B} \cdot \mathrm{I}$ oscillator ground state of ch.3.

$$
\mathrm{I}(\mathrm{h})>=\mathrm{U}(\mathrm{h}(\mathrm{g})) \mid 0>\quad \forall \mathrm{g} \in \mathrm{SL}(2, \mathrm{p})
$$

Any other element, $g^{\prime}$, of $S L(2, p)$ acts classicaly on the states $\mid z>$,

$$
U\left(g^{\prime}\right)|z(h)>=| z\left(h\left(g^{\prime} g\right)\right)>
$$

Because the metaplectic representation of SL(2,p) is irreducible, from (5.27) we find that the matrix,

$$
I=\frac{1}{N} \cdot \sum_{h \in S L(2, p) / O_{\mathbb{D}}(2)}|z(h)><z(h)|
$$

is the identity matrix, $I_{p x p}$, adjusting the normalization constant $\mathrm{N}$ (see chapter 4).

Any complex funciton $f$ on the Galois field GF[p], can be represented on the coherent states $\mathrm{l} z(\mathrm{~h})>$ using its components. The set of states $\mathrm{z}(\mathrm{h})>$ acts like an "orthonormal" system, although it is not:

$$
\left|f>=\frac{1}{N} \cdot \sum_{h}\right| z(h)><z(h) \mid f>
$$

Matrices or functions on GF[p $\left.\mathrm{p}^{2}\right]$ can also be represented on the coherent states $\mathrm{Iz}(\mathrm{h})>$ in an analogous manner with the HW coherent states (ch.4). 


\section{Table Capture}

In tables $I_{a-c}$, we give the primitive elements of $G F^{*}[p], O_{p}(2) \equiv C_{p}$ and $G F\left[p^{2}\right]$ for primes of the form $p=4 k-1 \leq 2003$. 


\section{Appendix}

In this appendix we summarize for completeness, some elementary facts about Galois fields $[7,16]$. The simplest Galois field is the set of numbers, ( $p$ prime number),

$$
\mathrm{GF}[\mathrm{p}]=\{0,1,2, \ldots, \mathrm{p}-1\}
$$

where all arithmetic operation are done modulo p.

The little Fermat theorem says that

$$
\mathrm{a}^{\mathrm{p}}=\mathrm{a} \bmod \mathrm{p} \quad \forall \mathrm{a} \in \mathrm{GF}[\mathrm{p}]
$$

and it follows that

$$
(\mathrm{a}+\mathrm{b})^{\mathrm{p}}=\mathrm{a}^{\mathrm{p}}+\mathrm{b}^{\mathrm{p}} \bmod \forall \mathrm{a}, \mathrm{b} \in \mathrm{GF}[\mathrm{p}]
$$

The multiplicative group of $\mathrm{GF}[\mathrm{p}], \mathrm{GF}^{*}[\mathrm{p}]$ :

$$
\mathrm{GF}^{*}[\mathrm{p}]=\{1,2, \ldots, \mathrm{p}-1\}
$$

is cyclic and there are primitive elements of period p-1, [18].

Now we pass over the representation theory of the group SL(2,p) the $2 \times 2$ integer matrices of unit determinat mod $\mathrm{p}[13,14]$.

The class representatives are given as

$$
1, z, a^{l}, b^{m}, c, d, z c, z d
$$

where

$$
1=\left(\begin{array}{ll}
1 & 0 \\
0 & 1
\end{array}\right), \quad z=-1, \quad a=\left(\begin{array}{cc}
g & 0 \\
0 & g^{-1}
\end{array}\right)
$$

g, a primitive element of GF[p] 
TABLE Ia

\begin{tabular}{|c|c|c|c|c|c|c|c|}
\hline $\begin{array}{c}4 \mathrm{k}-1 \\
\text { Prime } \\
\end{array}$ & $\mathrm{GF}[\mathrm{p}]$ & $\mathrm{C}_{\mathrm{p}}$ & $\mathrm{GF}\left[\mathrm{p}^{2}\right]$ & $\begin{array}{c}4 \mathrm{k}-1 \\
\text { Prime } \\
\end{array}$ & $\mathrm{GF}[\mathrm{p}]$ & $\mathrm{C}_{\mathrm{p}}$ & $G F\left[p^{2}\right]$ \\
\hline 3 & 2 & $0+I$ & $1+\mathrm{I}$ & 347 & 2 & $205+225 \mathrm{I}$ & $30+1811$ \\
\hline 7 & 3 & $2+5 I$ & $2+3 I$ & 359 & 7 & $154+112 \mathrm{I}$ & $2+196 \mathrm{I}$ \\
\hline 11 & 2 & $3+5 I$ & $2+3 I$ & 367 & 6 & $74+2211$ & $15+1911$ \\
\hline 19 & 2 & $16+12 I$ & $6+2 I$ & 379 & 2 & $304+228 \mathrm{I}$ & $175+68 I$ \\
\hline 23 & 5 & $19+10 I$ & $2+I$ & 383 & 5 & $307+154 \mathrm{I}$ & $2+I$ \\
\hline 31 & 3 & $10+26 \mathrm{I}$ & $1+8 I$ & 419 & 2 & $272+173 I$ & $86+2311$ \\
\hline 43 & 3 & $35+18 \mathrm{I}$ & $21+11 \mathrm{I}$ & 431 & 7 & $153+254 \mathrm{I}$ & $216+54 I$ \\
\hline 47 & 5 & $10+29 I$ & $2+1$ & 439 & 15 & $183+118 I$ & $56+55 I$ \\
\hline 59 & 2 & $48+361$ & $7+22 I$ & 443 & 2 & $355+178 \mathrm{I}$ & $26+395 I$ \\
\hline 67 & 2 & $14+41 \mathrm{I}$ & $22+11 I$ & 463 & 3 & $371+186 \mathrm{I}$ & $133+298 \mathrm{I}$ \\
\hline 71 & 7 & $5 l+13 I$ & $18+311$ & 467 & 2 & $94+2811$ & $409+29 I$ \\
\hline 79 & 3 & $65+11 I$ & $39+33 I$ & 479 & 13 & $339+85 I$ & $343+445 I$ \\
\hline 83 & 2 & $3+65 I$ & $2+9 I$ & 487 & 3 & $98+293 I$ & $187+337 \mathrm{I}$ \\
\hline 103 & 5 & $83+42 I$ & $2+I$ & 491 & 2 & $152+227 I$ & $251+4431$ \\
\hline 107 & 2 & $22+65 I$ & $68+73 I$ & 499 & 7 & $400+300 \mathrm{I}$ & $374+208 \mathrm{I}$ \\
\hline 127 & 3 & $26+77 I$ & $72+36 I$ & 503 & 5 & $368+313 I$ & $258+43 I$ \\
\hline 131 & 2 & $27+531$ & $40+57 \mathrm{I}$ & 523 & 2 & $419+210 \mathrm{I}$ & $233+145 \mathrm{I}$ \\
\hline 139 & 2 & $99+25 I$ & $10+72 I$ & 547 & 2 & $110+3291$ & $320+3871$ \\
\hline 151 & 6 & $59+12 I$ & $28+66 \mathrm{I}$ & 563 & 2 & $451+226 I$ & $511+26 I$ \\
\hline 163 & 2 & $131+66 I$ & $28+1419$ & 571 & 3 & $115+229 \mathrm{I}$ & $60+551 I$ \\
\hline 167 & 5 & $34+1011$ & $2+I$ & 587 & 2 & $112+508 \mathrm{I}$ & $88+181 \mathrm{I}$ \\
\hline 179 & 2 & $144+108 \mathrm{I}$ & $18+6 I$ & 599 & 7 & $109+10 \mathrm{I}$ & $76+102 I$ \\
\hline 191 & 19 & $130+181 I$ & $24+4 I$ & 607 & 3 & $122+365 I$ & $54+27 I$ \\
\hline 199 & 3 & $87+81 \mathrm{I}$ & $27+95 \mathrm{I}$ & 619 & 2 & $496+372 I$ & $328+971$ \\
\hline 211 & 2 & $43+85 I$ & $39+198 I$ & 631 & 3 & $98+146 \mathrm{I}$ & $298+312 \mathrm{I}$ \\
\hline 223 & 3 & $179+90 \mathrm{I}$ & $50+25 \mathrm{I}$ & 643 & 11 & $515+2581$ & $59+292 I$ \\
\hline 227 & 2 & $46+137 I$ & $71+78 I$ & 647 & 5 & $130+389 \mathrm{I}$ & $2+I$ \\
\hline 239 & 7 & $12+151 \mathrm{I}$ & $72+9 I$ & 659 & 2 & $138+6071$ & $46+2591$ \\
\hline 251 & 6 & $51+1011$ & $77+1391$ & 683 & 5 & $547+274 I$ & $2+682 I$ \\
\hline 263 & 5 & $211+106 \mathrm{I}$ & $2+I$ & 691 & 3 & $139+2771$ & $66+6691$ \\
\hline 271 & 6 & $126+188 I$ & $129+80 \mathrm{I}$ & 719 & 11 & $593+508 \mathrm{I}$ & $329+262 \mathrm{I}$ \\
\hline 283 & 3 & $227+114 \mathrm{I}$ & $25+129 \mathrm{I}$ & 727 & 5 & $146+437 I$ & $2+\mathrm{I}$ \\
\hline 307 & 5 & $293+135 I$ & $11+240 I$ & 739 & 3 & $592+444 \mathrm{I}$ & $93+708 I$ \\
\hline 311 & 17 & $257+220 \mathrm{I}$ & $4+I$ & 743 & 5 & $595+298 \mathrm{I}$ & $2+1$ \\
\hline 331 & 3 & $67+133 I$ & $226+35 \mathrm{I}$ & 751 & 3 & $531+133 I$ & $188+47 I$ \\
\hline
\end{tabular}


For $\mathrm{p}=4 \mathrm{k}+1$ we check that -1 , is a qres with square root,

$$
\mathrm{x}_{+}=\left(\frac{\mathrm{p}-1}{2}\right) !
$$

The Gausss quadratic sum is very useful and it is known in closed form, $[7,12,16,21]$

$$
\frac{1}{\sqrt{p}} \sum_{n=0}^{\mathrm{p}-1} \mathrm{e}^{-\frac{2 \pi \mathrm{i}}{\mathrm{p}} \cdot \mathrm{a} \cdot \mathrm{n}^{2}}=(\mathrm{alp})\left\{\begin{array}{l}
1 \\
n
\end{array}\right\} \begin{aligned}
& \mathrm{p}=4 \mathrm{k}+1 \\
& \mathrm{p}=4 \mathrm{k}-1
\end{aligned}, \quad \forall \mathrm{a} \in \mathrm{GF}^{*}[\mathrm{p}]
$$

The basic additive and multiplicative functions on GF[p] are the characters of the corresponding groups of $G F[p], G F+[p]$ and $G F^{*}[p],\left(\omega=e^{2 \pi i / p}\right),[16]$ :

$$
\begin{aligned}
& \chi_{j}(a)=\omega_{p}^{j \cdot a} \quad j=0,1, \ldots, p-1 \quad, \quad a \in G F[p] \\
& \pi_{j}(a)=\omega_{p-1}^{j \cdot n} \quad j=1, \ldots, p-2, \quad a=g^{n} \in G F^{*}[p]
\end{aligned}
$$

We can extend $\pi_{j}$ on GF[p]

$$
\begin{aligned}
& \pi_{j}(0)=0 \\
& \pi_{0}=\left[\begin{array}{c}
1 \\
0 \\
\ldots \\
0
\end{array}\right]
\end{aligned}
$$

These are orthogonal and complete systems of functions on GF[p].

We close the appendix by giving details of how construct the generator $R_{0}$, of the $O_{p}(2)$ subgroup of $\operatorname{SL}(2, \mathrm{p})$ :

$$
O_{p}(2)=\left\{\left(\begin{array}{cc}
a & b \\
-b & a
\end{array}\right) \mid a^{2}+b^{2}=1 \bmod p\right\}
$$


TABLE Ib

\begin{tabular}{|c|c|c|c|c|c|c|c|}
\hline $\begin{array}{c}4 \mathrm{k}-1 \\
\text { Prime }\end{array}$ & $G F[p]$ & $\mathrm{C}_{\mathrm{p}}$ & $\mathrm{GF}\left[\mathrm{p}^{2}\right]$ & $\begin{array}{c}\text { 4k-1 } \\
\text { Prime } \\
\end{array}$ & $G F[p]$ & $C_{p}$ & $\mathrm{GF}\left[\mathrm{p}^{2}\right]$ \\
\hline 787 & 2 & $158+4731$ & $305+2411$ & 1291 & 2 & $259+517 I$ & $538+2511$ \\
\hline 811 & 3 & $163+325 I$ & $559+84 I$ & 1303 & 6 & $1043+522 \mathrm{I}$ & $167+735 \mathrm{I}$ \\
\hline 823 & 3 & $659+330 \mathrm{I}$ & $195+509 \mathrm{I}$ & 1307 & 2 & $262+785 \mathrm{I}$ & $704+955 \mathrm{I}$ \\
\hline 827 & 2 & $166+497 \mathrm{I}$ & $267+280 \mathrm{I}$ & 1319 & 13 & $544+466 I$ & $317+409 I$ \\
\hline 839 & 11 & $297+494 I$ & $259+694 \mathrm{I}$ & 1327 & 3 & $266+797 I$ & $844+422 I$ \\
\hline 859 & 2 & $688+516 \mathrm{I}$ & $160+233 I$ & 1367 & 5 & $274+8211$ & $2+I$ \\
\hline 863 & 5 & $532+797 I$ & $42+682 I$ & 1399 & 13 & $1153+988 I$ & $712+178 I$ \\
\hline 883 & 2 & $707+354 \mathrm{I}$ & $83+400 I$ & 1423 & 3 & $1139+5701$ & $986+493 I$ \\
\hline 887 & 5 & $178+5331$ & $2+I$ & 1427 & 2 & $286+857 I$ & $487+470 \mathrm{I}$ \\
\hline 907 & 2 & $182+545 I$ & $75+416 \mathrm{I}$ & 1439 & 7 & $130+1330 I$ & $472+519 \mathrm{I}$ \\
\hline 911 & 17 & $376+3221$ & $4+I$ & 1447 & 3 & $290+869 I$ & $1184+592 \mathrm{I}$ \\
\hline 919 & 7 & $299+50 \mathrm{I}$ & $764+179 I$ & 1451 & 2 & $1099+667 \mathrm{I}$ & $946+326 I$ \\
\hline 947 & 2 & $692+589 \mathrm{I}$ & $96+931 \mathrm{I}$ & 1459 & 3 & $1168+876 I$ & $32+962 I$ \\
\hline 967 & 5 & $194+5811$ & $2+\mathrm{I}$ & 1471 & 6 & $793+453 I$ & $445+891$ \\
\hline 971 & 6 & $195+389 I$ & $215+719 I$ & 1483 & 2 & $1187+594 I$ & $1173+155 I$ \\
\hline 983 & 5 & $787+394 \mathrm{I}$ & $2+I$ & 1487 & 5 & $298+893 I$ & $2+I$ \\
\hline 991 & 6 & $817+700 I$ & $268+67 I$ & 1499 & 2 & $1059+265 I$ & $84+211$ \\
\hline 1019 & 2 & $717+799 I$ & $471+4311$ & 1511 & 2 & $491+821$ & $847+393 I$ \\
\hline 1031 & 14 & $196+892 I$ & $506+428 \mathrm{I}$ & 1523 & 2 & $1030+1441 \mathrm{I}$ & $736+385 I$ \\
\hline 1039 & 3 & $77+355 \mathrm{I}$ & $34+581 \mathrm{I}$ & 1531 & 2 & $307+6131$ & $105+1496 \mathrm{I}$ \\
\hline 1051 & 7 & $211+4211$ & $101+667 I$ & 1543 & 5 & $1235+618 I$ & $2+I$ \\
\hline 1063 & 3 & $851+426 \mathrm{I}$ & $224+112 I$ & 1559 & 19 & $276+459 I$ & $942+1015 \mathrm{I}$ \\
\hline 1087 & 3 & $218+653 I$ & $568+284 I$ & 1567 & 3 & $1107+277 I$ & $901+6171$ \\
\hline 1091 & 2 & $219+437 I$ & $619+570 \mathrm{I}$ & 1571 & 2 & $315+629 \mathrm{I}$ & $681+2271$ \\
\hline 1103 & 5 & $274+5791$ & $364+846 \mathrm{I}$ & 1579 & 3 & $1264+948 \mathrm{I}$ & $1101+1212 I$ \\
\hline 1123 & 2 & $899+450 \mathrm{I}$ & $30+1108 \mathrm{I}$ & 1583 & 5 & $1267+634 \mathrm{I}$ & $2+I$ \\
\hline 1151 & 17 & $178+266 I$ & $349+3001$ & 1607 & 5 & $322+965 \mathrm{I}$ & $2+I$ \\
\hline 1163 & 5 & $931+466 \mathrm{I}$ & $2+1162 I$ & 1619 & 2 & $1072+1146 \mathrm{I}$ & $872+1510 \mathrm{I}$ \\
\hline 1171 & 2 & $235+469 I$ & $98+748 I$ & 1627 & 3 & $326+9771$ & $1323+152 I$ \\
\hline 1187 & 2 & $238+713 I$ & $837+175 I$ & 1663 & 3 & $1331+666 \mathrm{I}$ & $488+244 \mathrm{I}$ \\
\hline 1223 & 5 & $34+1025 \mathrm{I}$ & $1085+1200 \mathrm{I}$ & 1667 & 2 & $1155+898 \mathrm{I}$ & $34+660 \mathrm{I}$ \\
\hline 1231 & 3 & $725+797 I$ & $1198+915 \mathrm{I}$ & 1699 & 3 & $1360+1020 \mathrm{I}$ & $1558+47 I$ \\
\hline 1259 & 2 & $1008+7561$ & $461+993 I$ & 1723 & 3 & $1379+690 \mathrm{I}$ & $718+1364 \mathrm{I}$ \\
\hline 1279 & 3 & $788+1181 \mathrm{I}$ & $1001+456 \mathrm{I}$ & 1747 & 2 & $350+1049 \mathrm{I}$ & $1261+243 I$ \\
\hline 1283 & 2 & $1027+514 I$ & $574+996 \mathrm{I}$ & 1759 & 6 & $1237+1379 I$ & $1745+1175 I$ \\
\hline
\end{tabular}




\section{LITERATURE}

[1] Y. Nambu, "Field theory of Galois Fields", in E.S. Fradkin Festschrift Symposium 1989; L. Brekke and P.G.O. Freund, "P-adic numbers in physics", Physics Rep. 233 (1993) 1; M. Waldschmidt, P. Moussa, J.-M. Luck and C. Itzykson, "From Number theory to Physics", (Les Houches, 1989), Springer Verlag Berlin Heidelberg 1992.

[2] P. Freund and M. Olson, Phys. Lett. 199B (1987) 186; P. Freund and E. Witten, Phys. Lett 199B (1987) 191; H. Frampton and Y. Okada, Phys. Rev. Lett. 60 (1988) 484; B. Grossman, Phys. Lett. 197B (1987) 101; Y. Meurice, I.J.M. Ph. A $\underline{4}$ (1989) 2211; I. Volovich, Class Quant. Grav. 4 (1987).

[3] G’t Hooft, J. State Phys. $\underline{53}$ (1988) 323; Nucl. Phys. B342 (1990) 471; G’t Hooft, K. Isler and S. Kalitzin, "Quantum Field Theoretic Behaviour of a Deterministic Cellular Automation”, Ultrecht Univ. Preprint, THU-92/7.

[4] J. Hannay and M. Berry, Physica 1D, 267 (1980).

[5] For a more recent discussion see :

P. Leboeuf and A. Voros, "Quantum Nodal points as fingerprints of Classical chaos", to appear in "Quantum Chaos", Ed. G. Casati and B.V. Chirikov, Cambridge Univ. Press 1993.

[6] C.K. Chui, "An introduction to Wavelets", Vol. I, II, Academic Press Inc. 1992.

[7] T. Apostol, "Introduction to Analytic Number Theory", Springer-Verlag N.Y. 1984; J.P. Serret," "Cours d' arithmétique”, PUF, $3^{\text {me }}$ ed. 1988.

[8] H. Weyl, "The theory of groups and quantum mechanics", Dover N.Y. 1931.

[9] J. Schwinger, Proc. of Nat of cad. Sc. 46 (1960) 257; 574; 883.

[10] For a recent discussion see :

J. Zak, Phys. Rev. B39 (1989) 694; R. Rammal and J. Bellissard, J. Phys. France 51 (1990) 1803.

[11] A. Connes, "Geometric non commutative", Inter Editions 1990, Paris. 
TABLE IC

\begin{tabular}{|c|c|c|c|c|c|c|c|}
\hline $\begin{array}{c}4 k-1 \\
\text { Prime }\end{array}$ & GF $[p]$ & $C_{p}$ & GF $\left[p^{2}\right]$ & $\begin{array}{c}4 k-1 \\
\text { Prime }\end{array}$ & GF $[p]$ & $C_{p}$ & GF $\left[p^{2}\right]$ \\
\hline 1783 & 10 & $1427+714 \mathrm{I}$ & $1467+1625 \mathrm{I}$ & 1879 & 6 & $290+434 \mathrm{I}$ & $1510+302 \mathrm{I}$ \\
\hline 1787 & 2 & $1489+1210 \mathrm{I}$ & $202+236 \mathrm{I}$ & 1907 & 2 & $1347+337 \mathrm{I}$ & $742+1139 \mathrm{I}$ \\
\hline 1811 & 6 & $363+725 \mathrm{I}$ & $955+922 \mathrm{I}$ & 1931 & 2 & $387+773 \mathrm{I}$ & $746+1536 \mathrm{I}$ \\
\hline 1823 & 5 & $1459+730 \mathrm{I}$ & $2+\mathrm{I}$ & 1951 & 3 & $572+714 \mathrm{I}$ & $838+1177 \mathrm{I}$ \\
\hline 1831 & 3 & $324+539 \mathrm{I}$ & $1016+2541 \mathrm{I}$ & 1979 & 2 & $1371+1066 \mathrm{I}$ & $453+1287 \mathrm{I}$ \\
\hline 1847 & 5 & $370+1109 \mathrm{I}$ & $2+\mathrm{I}$ & 1987 & 2 & $398+1193 \mathrm{I}$ & $111+938 \mathrm{I}$ \\
\hline 1867 & 2 & $374+1121 \mathrm{I}$ & $1583+142 \mathrm{I}$ & 1999 & 3 & $824+706 \mathrm{I}$ & $168+42 \mathrm{I}$ \\
\hline 1871 & 14 & $1370+776 \mathrm{I}$ & $392+1083 \mathrm{I}$ & 2003 & 5 & $1603+802 \mathrm{I}$ & $2001+\mathrm{I}$ \\
\hline
\end{tabular}


[26] I.M. Gelfand and M.A. Naimark, Dokl. Akad. Nank SSSR 55 (1947) 570; E.W. Aslassken and J.R. Klauder, J. Math. Phys. 9 (1968) 206, J. Math. Phys. 10 (1960) 2267; S. Lang, "SL (2R)" Addisson-Wesley, Reading MA 1975.

[27] V.F. Molchanov, Siberian Mathematical Journal 18 (1977) 174; Functional Analysis and its Appl. 14 (1980) 73.

[28] T. Paul, J. Math. Phys. 25 (1984) 3252.

[29] S.G. Mallat, Trans. Amer. Math. Soc. $\underline{315}$ (1989) 69.

[30] "Wavelets on Discrete fields", K. Flornes, A. Grossmann, M. Holschneider and B. Torrésani, CPT-93/p.2932, CNRS Luminy prepring.

[31] O. Martin, A.M. Odlyzko and S. Wolfram, Comm. Math. Physics 93 (1984) 219.

[32] L.E. Reichl, "The transition to chaos" Springel Verlag 1992 N. York. 